\title{
Quantitative analysis of total $\beta$-subunit of human chorionic gonadotropin concentration in urine by immunomagnetic reduction to assist in the diagnosis of ectopic pregnancy
}

\author{
This article was published in the following Dove Press journal: \\ International Journal of Nanomedicine \\ 30 March 2015 \\ Number of times this article has been viewed
}

\section{Chen-Yu Chen ${ }^{1-3}$ \\ Yuh-Ming $\mathrm{Hwu}^{2}$ \\ Chie-Pein Chen ${ }^{2}$ \\ Chia-Chen Chang ${ }^{4}$}

'Department of Medicine, Mackay Medical College, New Taipei City, Taiwan; ' ${ }^{2}$ epartment of Obstetrics and Gynecology, Mackay Memorial Hospital, Taipei, Taiwan; ${ }^{3}$ Mackay Junior College of Medicine, Nursing, and Management, Taipei, Taiwan; ${ }^{4}$ Institute of Biomedical Engineering, National Taiwan University, Taipei, Taiwan
Correspondence: Chen-Yu Chen Department of Obstetrics and Gynecology, Mackay Memorial Hospital, No 92, Section 2, Zhong-Shan North Road, Taipei 10449, Taiwan

Tel +886225433535

Fax +886225433642

Email fI2248I@mmh.org.tw
Background: The initial diagnosis of ectopic pregnancy depends on physical examination, ultrasound, and serial measurements of total $\beta$-subunit of human chorionic gonadotropin (hCG $\beta$ ) concentrations in serum. The aim of this study was to explore the possibility of using quantitative analysis of total hCG $\beta$ in urine rather than in serum by immunomagnetic reduction (IMR) assay as an alternative method to diagnose an ectopic pregnancy.

Methods: We established a standard calibration curve of IMR intensity against total hCG $\beta$ concentration based on standard hCG $\beta$ samples, and used an IMR assay to detect total hCG $\beta$ concentrations in the urine of pregnant women with lower abdominal pain and/or vaginal bleeding. The final diagnosis of ectopic pregnancy was based on ultrasound scans, operative findings, and pathology reports. In this prospective study, ten clinical samples were used to analyze the relationship of total hCG $\beta$ IMR signals between urine and serum. Furthermore, 20 clinical samples were used to analyze the relationship between urine IMR signals and serum levels of total hCG $\beta$.

Results: The calibration curve extended from $0.01 \mathrm{ng} / \mathrm{mL}$ to $10,000 \mathrm{ng} / \mathrm{mL}$ with an excellent correlation $\left(R^{2}=0.999\right)$. In addition, an excellent correlation of total hCG $\beta$ IMR signals between urine and serum was noted $\left(R^{2}=0.994\right)$. Furthermore, a high correlation between urine IMR signals and serum levels of total hCG $\beta$ was noted $\left(R^{2}=0.862\right)$.

Conclusion: An IMR assay can quantitatively analyze total hCG $\beta$ concentrations in urine, and is a potential candidate for point-of-care testing to assist in the diagnosis of ectopic pregnancy.

Keywords: beta-subunit of human chorionic gonadotropin, immunomagnetic reduction, ectopic pregnancy, point-of-care

\section{Introduction}

Ectopic pregnancy is the leading cause of maternal mortality in the first trimester, and has been reported to cause $4 \%-10 \%$ of pregnancy-related mortality. ${ }^{1}$ It occurs in $1 \%-2 \%$ of all pregnancies, and the incidence has increased over the past two decades. ${ }^{2,3}$ Early detection of ectopic pregnancy is not easy because the initial signs and symptoms, such as a lack of menstrual period, low abdominal pain, and vaginal bleeding, are often ambiguous, and may be seen in a normal pregnancy. Thus, the initial detection of ectopic pregnancy in the first trimester remains a major challenge for clinicians. The diagnosis of ectopic pregnancy is primarily determined through transvaginal ultrasound and serial serum human chorionic gonadotropin (hCG) levels. ${ }^{4}$

hCG is a member of the glycoprotein hormone family, and has a molecular weight of approximately 46,000 Daltons. After conception, hCG is initially synthesized by the 
trophoblastic cells of the placenta and maintains the corpus luteum. ${ }^{5}$ The prompt increase in serum hCG levels makes it an ideal biomarker for the early detection of pregnancy. Similar to other glycoprotein hormones such as the follicle stimulating hormone, luteinizing hormone, and thyroid stimulating hormone, hCG consists of two noncovalently bound subunits, $\alpha$ and $\beta$. The $\alpha$ subunit of hCG consists of 92 amino acids, and the $\beta$ subunit of hCG (hCG $\beta$ ) consists of 145 amino acids. The structure of the $\alpha$ subunit is essentially identical for the transactivation of receptors, whereas the structure of the $\beta$ subunit is transcribed from separate genes and confers the biological and immunological specificity of receptors. ${ }^{6}$ Previous studies have revealed that total hCG $\beta$ levels can be useful in detecting spontaneous abortions and ectopic pregnancies. ${ }^{7-9}$ Further applications have been demonstrated for hCG or hCG $\beta$ measurements in determining the risk for fetal Down syndrome, maternal preeclampsia, gestational trophoblastic diseases, and germ cell malignancies. ${ }^{10-12}$

Serum or urine total hCG $\beta$ levels are detectable in $75 \%$ of pregnancies by the 9th day after ovulation and more than $95 \%$ by the 10 th day after ovulation. ${ }^{13}$ At the point of missed menses, the serum total hCG $\beta$ levels are normally about $100 \mathrm{mIU} / \mathrm{mL}$. The serum total hCG $\beta$ levels reach a peak value at around $100,000 \mathrm{mIU} / \mathrm{mL}$ by 8 weeks to 10 weeks of gestation, and then decrease and remain stable at about $20,000 \mathrm{mIU} / \mathrm{mL}$ throughout the rest of the pregnancy. ${ }^{14}$

The doubling time of the total hCG $\beta$ level is 1.4-2.1 days in normal early pregnancy. ${ }^{15}$ The serum total hCG $\beta$ level rises by more than $66 \%$ every 48 hours in $85 \%$ of intrauterine pregnancies. Thus, a rise in the total hCG $\beta$ level of less than $66 \%$ suggests the possibility of ectopic pregnancy, while a fall by more than $50 \%$ strongly suggests the possibility of a spontaneous abortion. ${ }^{89}$ A single total hCG $\beta$ level is also clinically useful in determining whether or not the pregnancy is intrauterine. Ectopic pregnancy should be considered if no intrauterine gestational sac can be found at a serum total hCG $\beta$ level of more than $1,500 \mathrm{mIU} / \mathrm{mL}$ or $2,000 \mathrm{mIU} / \mathrm{mL}$ on a transvaginal ultrasound scan, or more than $6,500 \mathrm{mIU} / \mathrm{mL}$ on a transabdominal ultrasound scan. ${ }^{16,17}$ Various quantitative methods have been used to measure the concentrations of biomolecules in medical laboratories since the 1960s, of which enzyme-linked immunosorbent assay (ELISA) is the best known. ${ }^{18}$ The subsequent development of chemiluminescence immunoassay (CLIA) provided a detection technique with increased sensitivity and specificity after the 1970 s. ${ }^{19}$ Nevertheless, there are several drawbacks with these methods, such as the extra time required for washing and incubation between the addition of reagents, decay of color signal intensity, and the requirement of two different antibodies.
Immunomagnetic reduction (IMR) assay is a promising method to overcome these disadvantages, and it has been investigated for the quantitative detection of biomolecules by biofunctionalized magnetic nanoparticles. ${ }^{20,21}$ The use of quantitative measurements of serum total hCG $\beta$ concentrations to assist in the diagnosis of ectopic pregnancy is well established, but quantitative urine assays are not readily accessible. With regards to point-of-care testing, the development of quantitative urine total hCG $\beta$ assays for the diagnosis of ectopic pregnancy is essential. In this study, the feasibility of assaying urine total hCG $\beta$ concentrations using IMR was investigated. In addition to synthesizing the reagents for the IMR assay, the characteristics of assaying total hCG $\beta$ via IMR were explored. Furthermore, we performed a prospective study to detect urine total hCG $\beta$ concentrations of pregnant women by IMR assays, and attempted to verify the potential of the urine IMR assay for the diagnosis of ectopic pregnancy.

\section{Materials and methods Sample collection}

This prospective study was performed in Mackay Memorial Hospital, Taipei, Taiwan from April 2013 to November 2013. The serum and urine samples of pregnant women who complained of lower abdominal pain and/or vaginal bleeding in the first trimester were collected. We then sent the samples to the laboratory for IMR detection using an IMR analyzer (XacPro-E; MagQu, New Taipei, Taiwan). The IMR signals were measured in duplicate for each sample. The final diagnosis of ectopic pregnancy was based on ultrasound scans, operative findings, and pathology reports. This study was approved by the Mackay Memorial Hospital Institutional Review Board (IRB \#12MMHIS179), and informed consent was obtained from all participants for the collection and analysis of clinical samples.

\section{IMR mechanism}

Figure 1 illustrates the mechanism of IMR. Homogeneously magnetic nanoparticles, which are coated with hydrophilic surfactants and antibodies, are individually scattered in the solution. After the addition of external multiple alternating current (ac) magnetic fields, individual magnetic nanoparticles are induced to oscillate and rotate. Under external multiple ac magnetic fields, the magnetic solution displays a magnetic property termed as mixed-frequency ac magnetic susceptibility $\chi_{\mathrm{ac}}$. The value of $\chi_{\mathrm{ac}}$ determined before magnetic nanoparticles connect with the targeted biomarkers is expressed as $\chi_{\mathrm{ac}, \mathrm{o}}$. In this study, magnetic nanoparticles connected with the targeted hCG $\beta$ (ab96167; Abcam, Cambridge, MA, USA) via coating with anti-hCG $\beta$ antibodies (ab764; Abcam) on the 
A

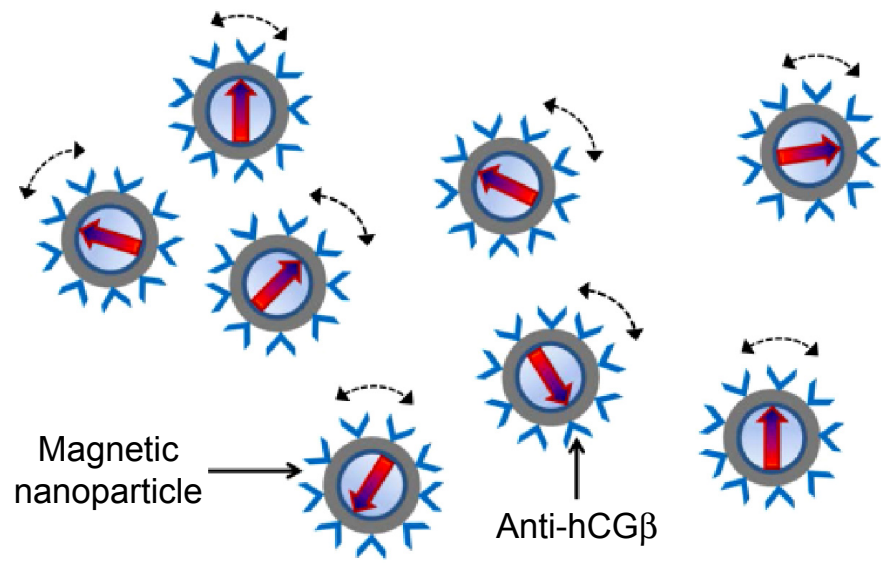

B
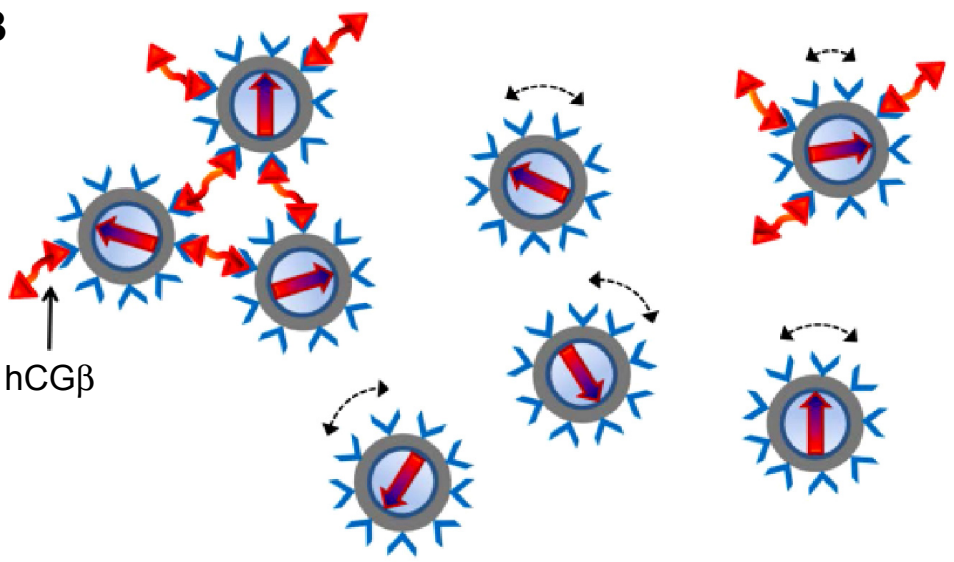

Figure I Illustration of the association between hCG $\beta$ biomarkers and magnetic nanoparticles coated with anti-hCG $\beta$ antibodies.

Notes: (A) Magnetic nanoparticles oscillate and rotate individually with the applied external multiple ac magnetic fields before binding with hCG $\beta$. (B) Magnetic nanoparticles become larger or clustered after binding with $\mathrm{hCG} \beta$, and thus oscillate and rotate much more slowly than the original individual magnetic nanoparticles.

Abbreviation: $h C G \beta$, total $\beta$-subunit of human chorionic gonadotropin.

shell surface, with the immune complexes becoming either larger or clustered. The larger/clustered magnetic nanoparticles oscillate and rotate much more slowly than the original individual magnetic nanoparticles as a result of physical limitation, which leads to a reduction in $\chi_{\mathrm{ac}}$. The reduced $\chi_{\mathrm{ac}}$ is expressed as $\chi_{\mathrm{ac}, \mathrm{b}}$, where $\phi$ refers to the concentration of the targeted biomarkers. In theory, a greater reduction in $\chi_{\mathrm{ac}}$ is anticipated if the solution contains higher concentrations of the targeted biomarkers, resulting in still larger/more clustered magnetic nanoparticles. The concentration of the targeted biomarkers was calculated as the reduction in $\chi_{\mathrm{ac}}$, and the IMR signal was acquired using the following equation:

$$
\operatorname{IMR}(\%)=\left(\chi_{\mathrm{ac}, \mathrm{o}}-\chi_{\mathrm{ac}, \phi}\right) / \chi_{\mathrm{ac}, \mathrm{o}} \times 100 \%
$$

\section{Preparation of magnetic reagents}

Magnetic $\mathrm{Fe}_{3} \mathrm{O}_{4}$ nanoparticles (MagQu) coated with dextran as a hydrophilic surfactant and anti-hCG $\beta$ antibodies (Figure 2A) were dispersed in phosphate buffered saline
(PBS) solution ( $\mathrm{pH}=7.2)$. Dextran was oxidized to generate aldehyde groups $(-\mathrm{CHO})$ to biofunctionalize the magnetic nanoparticles with antibodies against hCG $\beta$, and then it responded to anti-hCG $\beta$ with the dextran aldehyde groups to form a covalent bond $(-\mathrm{CH}=\mathrm{N}-)$, thus yielding the magnetic reagent to detect hCG $\beta$ concentrations..$^{22}$ The size distribution of the magnetic nanoparticles was analyzed by laser dynamic scattering, and the mean diameter was found to be $52.8 \mathrm{~nm}$ (Figure 2B). Through magnetic separation, unbound anti-hCG $\beta$ was detached from the solution, and anti-hCG $\beta$ biofunctionalized magnetic nanoparticles were disseminated in the PBS solution. The concentration of the magnetic reagent was $0.1 \mathrm{emu} / \mathrm{g}$ and it was stored at $4^{\circ} \mathrm{C}$.

\section{IMR measurements}

We used an ac magnetosusceptometer (XacPro-E; MagQu) to detect the IMR signals of the magnetic reagent caused by the association between the magnetic particles and hCG $\beta$ concentrations. First, we adjusted the magnetic reagent from $4^{\circ} \mathrm{C}$ to room temperature, and kept it at room temperature 


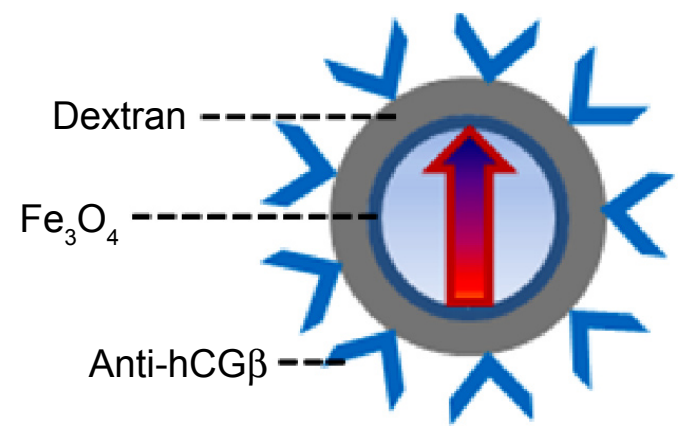

B

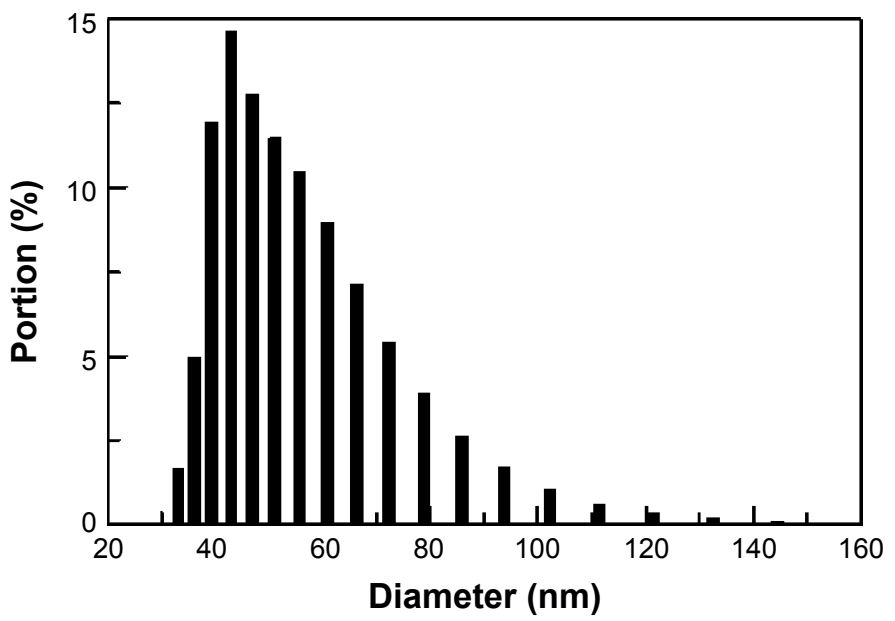

Figure 2 Illustration of magnetic nanoparticles.

Notes: (A) Magnetic iron oxide $\left(\mathrm{Fe}_{3} \mathrm{O}_{4}\right)$ nanoparticles coated with dextran and anti-hCG $\beta$ antibodies. (B) Statistics of magnetic nanoparticle diameters with the mean of $52.8 \mathrm{~nm}$.

Abbreviation: hCG $\beta$, total $\beta$-subunit of human chorionic gonadotropin.

(about $25^{\circ} \mathrm{C}$ ) for 5 minutes. We then used a vortex mixer to shake both the magnetic reagent and the urine/serum samples for 15 seconds. We next placed $40 \mu \mathrm{L}$ of the magnetic reagent into a glass tube, took $60 \mu \mathrm{L}$ of the clinical samples, and used the vortex mixer to shake the mixture for 15 seconds. Finally, we placed the mixture into the ac magnetosusceptometer for IMR measurements.

\section{Results}

\section{Real-time $\chi_{\mathrm{ac}}$ measurements}

An example of the real-time magnetic response $\chi_{\mathrm{ac}}$ of the mixture of the magnetic reagent and $1 \mathrm{ng} / \mathrm{mL}$ hCG $\beta$ solution is shown in Figure 3A. The data from 0 to 1.5 hours represent the magnetic response $\chi_{\mathrm{ac}, \mathrm{o}}$ of the mixture before incubation. The time-average value of $\chi_{\mathrm{ac}, \mathrm{o}}$ was 55.02. The data decreased from 1.5 hours to 4 hours, meaning that the magnetic nanoparticles were reacting with hCG $\beta$, and this was defined as the incubation period. After the reaction/incubation at room temperature (about $25^{\circ} \mathrm{C}$ ), the magnetic response $\chi_{\text {ac, } \phi}$ of the mixture approached another stable condition, as the data from 4 hours to 5 hours show. The time-average value was 54.44 . The significant reduction in magnetic response verified the conjugation between the magnetic nanoparticles and hCG $\beta$. Moreover, the IMR signal could be obtained as $\left(\chi_{\mathrm{ac}, \mathrm{o}}-\chi_{\mathrm{ac}, \mathrm{\phi}}\right) /$ $\chi_{\text {ac,o }} \times 100 \%=(55.02-54.44) / 55.02 \times 100 \%=1.05 \%$. After duplicating the IMR measurements (Figure 3B), the mean value and the standard deviation were $1.04 \%$ and $0.02 \%$, respectively, resulting in a coefficient of variation $(\mathrm{CV})$ of $1.92 \%$. The assay precision is calculated as CV. The low CV (less than 5\%) indicates that the IMR assay has good precision and reproducibility in measuring urine hCG $\beta$.

\section{Calibration curve establishment}

Figure 4 shows the IMR calibration curve established from duplicate measurements of hCG $\beta$ concentrations and ranged from $0.01 \mathrm{ng} / \mathrm{mL}$ to $10,000 \mathrm{ng} / \mathrm{mL}$. The signals of IMR (\%) were measured with hCG $\beta$ concentrations of $0.01 \mathrm{ng} / \mathrm{mL}$, $0.1 \mathrm{ng} / \mathrm{mL}, 1 \mathrm{ng} / \mathrm{mL}, 10 \mathrm{ng} / \mathrm{mL}, 100 \mathrm{ng} / \mathrm{mL}, 1,000 \mathrm{ng} / \mathrm{mL}$, and $10,000 \mathrm{ng} / \mathrm{mL}$ (Table 1). The data well fitted the fourparameter logistic equation IMR $(\%)=(A-B) /\left(1+\left[\phi_{\mathrm{hCG \beta}} /\right.\right.$ $\left.\left.\phi_{0}\right]^{3}\right)+B,{ }^{23}$ in which $\mathrm{A}$ is the minimal IMR signal, B is the maximal IMR signal, $\phi_{\mathrm{hCG} \beta}$ is the hCG $\beta$ concentration, $\phi_{\mathrm{o}}$ is the concentration of the inflection point, corresponding to the half maximal IMR signal, and $\gamma$ is the slope at the inflection point of the calibration curve. In this IMR calibration curve, the values were $A=0.75, B=1.92, \phi_{\mathrm{o}}=9.80$, and $\gamma=0.51$. These data showed excellent agreement when fitted to the calibration curve, with a coefficient of determination $\left(R^{2}\right)$ of 0.999 .

\section{IMR assay for urine and serum samples}

Ten clinical samples of the pregnant women were used to analyze the relationship of total hCG $\beta$ IMR signals between urine and serum (Table 2). Figure 5 shows that the IMR signal for hCG $\beta$ in urine is proportional to that in serum with excellent correlation $\left(R^{2}=0.994\right)$. The proportional constant was 1.12 , implying that the hCG $\beta$ concentration in urine is higher than that in serum.

\section{IMR assay for urine samples versus CLIA for serum samples}

Twenty clinical samples of the pregnant women were then obtained to analyze the relationship between urine hCG $\beta$ 
A

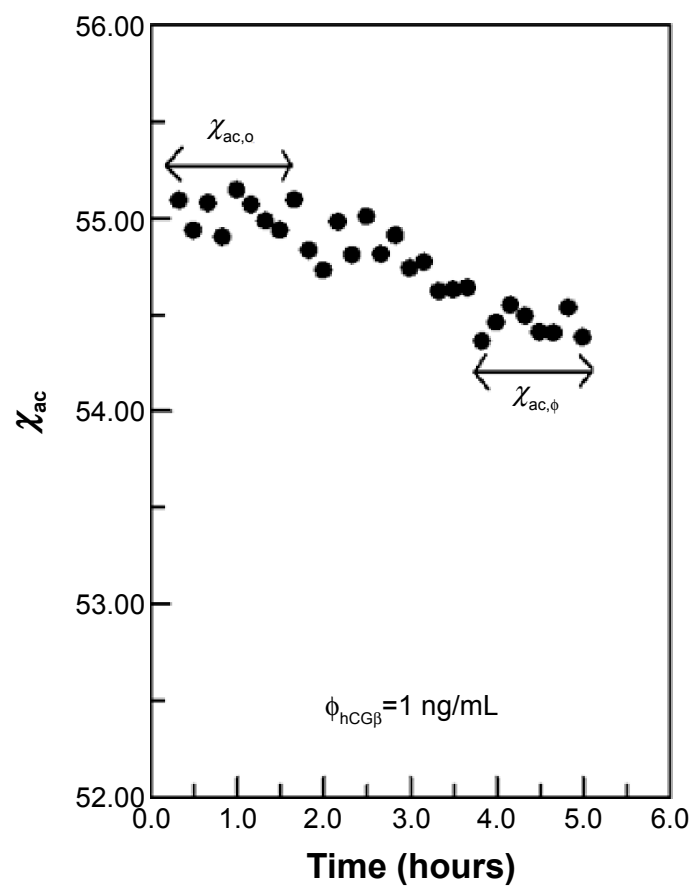

B

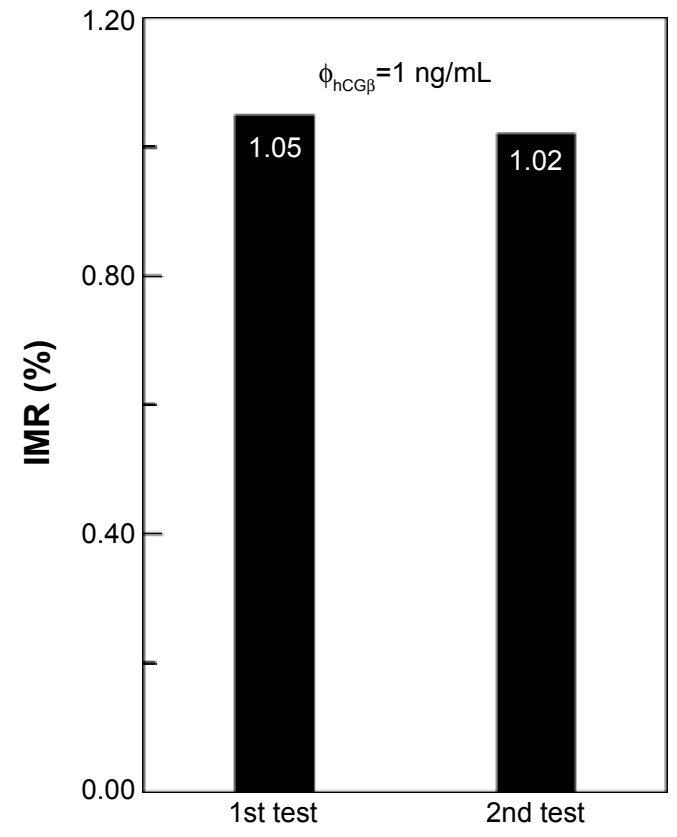

Figure 3 Immunomagnetic reduction assay of hCG $\beta$.

Notes: (A) Real-time $\chi_{\mathrm{ac}}$ signal of the magnetic reagent after being mixed with I $\mathrm{ng} / \mathrm{mL} \mathrm{hCG} \beta$ solution. (B) IMR signals for independent duplicate tests of I $\mathrm{ng} / \mathrm{mL}$ hCG $\beta$ solution.

Abbreviations: hCG $\beta$, total $\beta$-subunit of human chorionic gonadotropin; IMR, immunomagnetic reduction.

IMR signals and serum hCG $\beta$ CLIA values using an Access immunoassay analyzer (Beckman Coulter, Fullerton, CA, USA) (Table 3). Twelve of the 20 women were finally diagnosed with ectopic pregnancies (not including one case with tubal abortion proved by laparoscopy), five with intrauterine viable pregnancies, and three with spontaneous abortions (including one case with tubal abortion). The average hCG $\beta$ concentrations were much lower in the samples of the women with spontaneous abortions (366.6 mIU/mL) than in those of the women with ectopic pregnancies $(4,823.4 \mathrm{mIU} / \mathrm{mL})$ and viable intrauterine pregnancies $(6,105.8 \mathrm{mIU} / \mathrm{mL})$. The linear trend test was used to detect

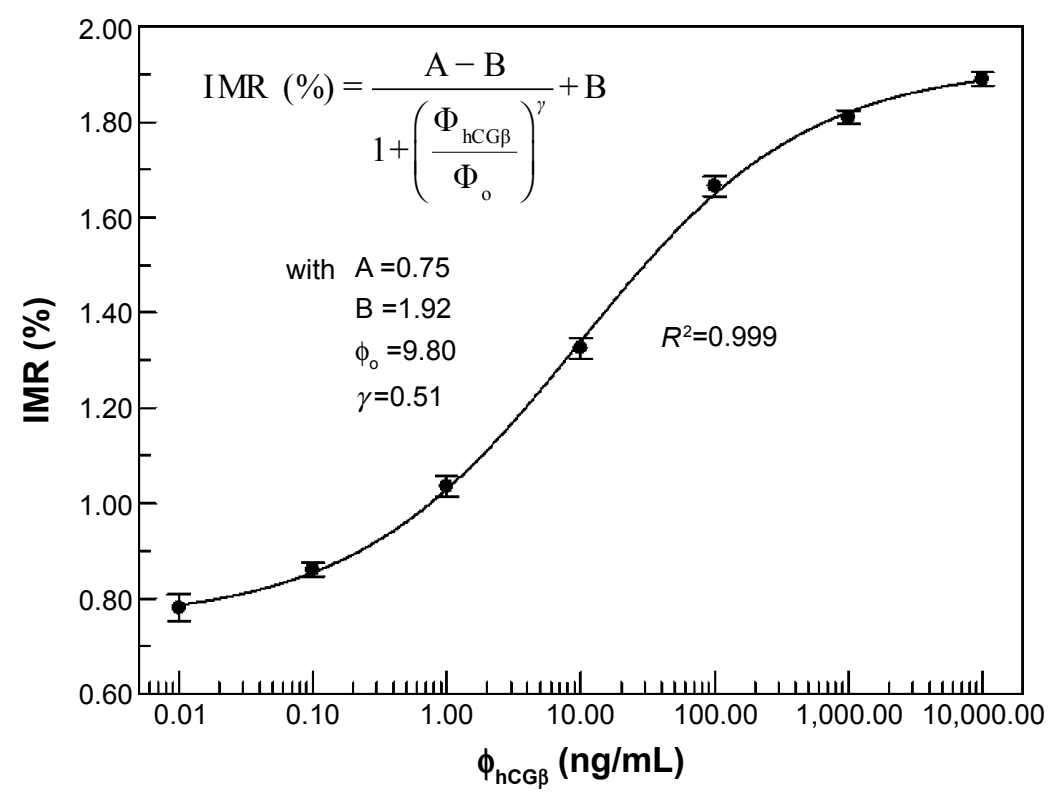

Figure 4 Calibration curve of IMR signals against hCG $\beta$ concentrations $\left(R^{2}=0.999\right)$.

Note: Points represent mean \pm standard deviation.

Abbreviations: hCG $\beta$, total $\beta$-subunit of human chorionic gonadotropin; IMR, immunomagnetic reduction. 
Table I The mean value, SD, and CV of hCG $\beta$ concentrationdependent IMR signals for the standard calibration curve

\begin{tabular}{llll}
\hline$\phi_{\mathrm{hCG} \beta}(\mathrm{ng} / \mathrm{mL})$ & Mean $(\%)$ & SD (\%) & CV (\%) \\
\hline $0.0 \mathrm{I}$ & 0.78 & 0.03 & 3.85 \\
0.1 & 0.86 & 0.014 & 1.63 \\
$\mathrm{I}$ & 1.04 & 0.02 & 1.92 \\
10 & 1.33 & 0.02 & 1.50 \\
100 & 1.67 & 0.02 & 1.20 \\
1,000 & 1.81 & 0.014 & 0.77 \\
10,000 & 1.89 & 0.014 & 0.74 \\
\hline
\end{tabular}

Abbreviations: hCG $\beta$, total $\beta$-subunit of human chorionic gonadotropin; IMR, immunomagnetic reduction; SD, standard deviation; $\mathrm{CV}$, coefficient of variation.

the hCG $\beta$ IMR signals in the three groups, and it followed the linear trend (means in abortion, ectopic pregnancy, and intrauterine pregnancy were $1.29,1.68$, and 1.72 , respectively) $(P=0.020)$. Furthermore, no correlation between hCG $\beta$ IMR signals and gestational weeks was noted by Pearson correlation analysis $(P=0.258)$, and thus the gestational week was not a confounding factor in this study. Figure 6 shows the experimental relationship between urine hCG $\beta$ IMR signals and serum hCG $\beta$ CLIA values. The urine hCG $\beta$ IMR signals were linear to the serum hCG $\beta$ CLIA values, with a slope of $7.32 \times 10^{-5}$ and an intercept in the IMR axis at 1.30. A high correlation between urine IMR signals and serum CLIA values of hCG $\beta$ was also noted $\left(R^{2}=0.862\right)$.

Table 2 The mean value, SD, and CV of urine and serum hCG $\beta$ concentration-dependent IMR signals

\begin{tabular}{lllll}
\hline Number & Sample type & Mean (\%) & SD (\%) & CV (\%) \\
\hline 1 & Urine & 1.03 & 0.035 & 3.45 \\
& Serum & 0.94 & 0.028 & 3.01 \\
2 & Urine & 2.23 & 0.028 & 1.27 \\
3 & Serum & 1.71 & 0.035 & 2.07 \\
& Urine & 1.08 & 0.035 & 3.29 \\
4 & Serum & 0.96 & 0.028 & 2.95 \\
& Urine & 2.38 & 0.049 & 2.08 \\
5 & Serum & 2.19 & 0.042 & 1.94 \\
& Urine & 1.21 & 0.007 & 0.59 \\
6 & Serum & 1.14 & 0.028 & 2.48 \\
& Urine & 1.13 & 0.028 & 2.5 \\
7 & Serum & 1.02 & 0.014 & 1.39 \\
& Urine & 1.86 & 0.014 & 0.76 \\
8 & Serum & 1.7 & 0.014 & 0.83 \\
& Urine & 1.24 & 0.021 & 1.72 \\
9 & Serum & 1.21 & 0.014 & 1.17 \\
& Urine & 1.35 & 0.014 & 1.05 \\
10 & Serum & 1.13 & 0.014 & 1.25 \\
& Urine & 1.58 & 0.042 & 2.69 \\
& Serum & 1.46 & 0.028 & 1.94 \\
\hline
\end{tabular}

Abbreviations: hCG $\beta$, total $\beta$-subunit of human chorionic gonadotropin; IMR, immunomagnetic reduction; SD, standard deviation; CV, coefficient of variation.

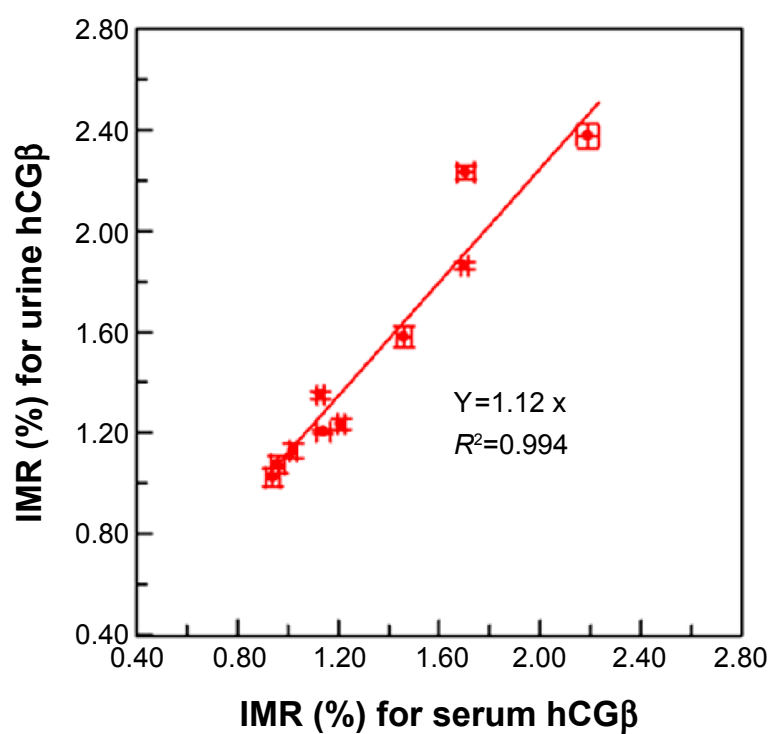

Figure 5 Relationship of total hCG $\beta$ IMR signals between urine and serum $\left(R^{2}=0.994\right)$.

Note: Points represent mean \pm standard deviation.

Abbreviations: hCG $\beta$, total $\beta$-subunit of human chorionic gonadotropin; IMR, immunomagnetic reduction.

\section{Discussion}

Ectopic pregnancy is an important cause of maternal morbidity and mortality worldwide. The diagnosis of ectopic pregnancy mainly relies on transvaginal ultrasound and serial serum hCG $\beta$ levels. ${ }^{4}$ However, there are limitations of the current diagnostic algorithms, and an initial misdiagnosis is still possible because of nonspecific symptoms and signs in the early stage of ectopic pregnancy. ${ }^{24}$ Increases in the sensitivity of ultrasound and hCG $\beta$ assays are crucial for the diagnosis of ectopic pregnancy and to reduce the necessity of surgery. Several factors have been reported to reduce the sensitivity of ultrasound, such as inexperienced ultrasonographers, obese patients, substantial absence of quantitative parameters, and unsatisfactory ultrasound resolution..$^{25,26}$ Furthermore, serial measurements of serum hCG $\beta$ levels over several days need to be performed in a hospital setting, leading to greater patient inconvenience and likely delaying the diagnosis of ectopic pregnancy. From this point of view, the development of quantitative urine total hCG $\beta$ assays to assist in the diagnosis of ectopic pregnancy is crucial for point-of-care testing.

Our study demonstrated an ideal lower limit of detection (LOD) of hCG $\beta$ concentration by IMR assays. In the logistic equation of the calibration curve, parameter A denoted the noise level for the IMR signal, where $\phi_{\mathrm{hCG}}$ was equal to 0 . The noise of the IMR assay primarily resulted from electronic interactions of the ac magnetosusceptometer. The LOD was defined as a concentration higher than the 
Table 3 Comparison of urine hCG $\beta$ IMR signals and serum hCG $\beta$ CLIA values in the clinical samples

\begin{tabular}{|c|c|c|c|c|c|c|}
\hline \multirow[t]{2}{*}{ Number } & \multicolumn{3}{|l|}{ IMR } & \multirow{2}{*}{$\begin{array}{l}\text { CLIA (mIU/ } \\
\mathrm{mL})\end{array}$} & \multirow{2}{*}{$\begin{array}{l}\text { Detection week } \\
\text { (week and day/7) }\end{array}$} & \multirow[t]{2}{*}{ Final diagnosis } \\
\hline & Mean (\%) & SD (\%) & CV (\%) & & & \\
\hline I & 1.58 & 0.042 & 2.69 & 3,665 & $80 / 7$ & Ectopic pregnancy \\
\hline 2 & $\mathrm{I} .43$ & 0.014 & 0.99 & 2,867 & $56 / 7$ & Ectopic pregnancy \\
\hline 3 & 1.32 & 0.021 & 1.61 & 437.3 & $81 / 7$ & Abortion \\
\hline 4 & 1.2 & 0.014 & 1.18 & 185.1 & $53 / 7$ & Tubal abortion \\
\hline 5 & 1.86 & 0.014 & 0.76 & 9,353 & $55 / 7$ & Intrauterine pregnancy \\
\hline 6 & 1.35 & 0.014 & 1.05 & 477.3 & $64 / 7$ & Abortion \\
\hline 7 & 2.00 & 0.049 & 2.48 & 11,343 & $6 \mathrm{I} / 7$ & Intrauterine pregnancy \\
\hline 8 & 1.54 & 0.007 & 0.46 & 1,846 & $70 / 7$ & Ectopic pregnancy \\
\hline 9 & 1.59 & 0.007 & 0.45 & 2,291 & $46 / 7$ & Intrauterine pregnancy \\
\hline 10 & 1.49 & 0.007 & 0.48 & 3,292 & $64 / 7$ & Ectopic pregnancy \\
\hline II & 1.43 & 0.014 & 0.99 & 3,225 & $70 / 7$ & Ectopic pregnancy \\
\hline 12 & 1.68 & 0.021 & 1.27 & 3,992 & $53 / 7$ & Intrauterine pregnancy \\
\hline 13 & 1.53 & 0.042 & 2.77 & 3,018 & $86 / 7$ & Ectopic pregnancy \\
\hline 14 & 1.46 & 0.014 & 0.97 & 3,550 & $5 \mathrm{I} / 7$ & Intrauterine pregnancy \\
\hline 15 & 1.79 & 0.014 & 0.79 & 6,534 & $63 / 7$ & Ectopic pregnancy \\
\hline 16 & 1.96 & 0.049 & 2.53 & 7,552 & $44 / 7$ & Ectopic pregnancy \\
\hline 17 & 2.06 & $0.05 I$ & 2.49 & 8,506 & $65 / 7$ & Ectopic pregnancy \\
\hline 18 & 1.65 & 0.021 & 1.29 & 4,512 & $65 / 7$ & Ectopic pregnancy \\
\hline 19 & 1.83 & 0.014 & 0.77 & 5,843 & $64 / 7$ & Ectopic pregnancy \\
\hline 20 & 1.87 & 0.007 & 0.38 & 7,021 & $46 / 7$ & Ectopic pregnancy \\
\hline
\end{tabular}

Abbreviations: hCG $\beta$, total $\beta$-subunit of human chorionic gonadotropin; IMR, immunomagnetic reduction; CLIA, chemiluminescence immunoassay; SD, standard deviation; $\mathrm{CV}$, coefficient of variation.

noise level by two standard deviations for IMR signals at the lowest detectable concentration. ${ }^{27}$ The standard deviation for IMR at the lowest concentration $(0.01 \mathrm{ng} / \mathrm{mL})$ was found to be $0.03 \%$, and therefore, the IMR signal of LOD was $0.81 \%$. Via the logistic equation, the LOD for assaying hCG $\beta$ using IMR was $0.03 \mathrm{ng} / \mathrm{mL}$, and is equal to $0.36 \mathrm{mIU} / \mathrm{mL}$.

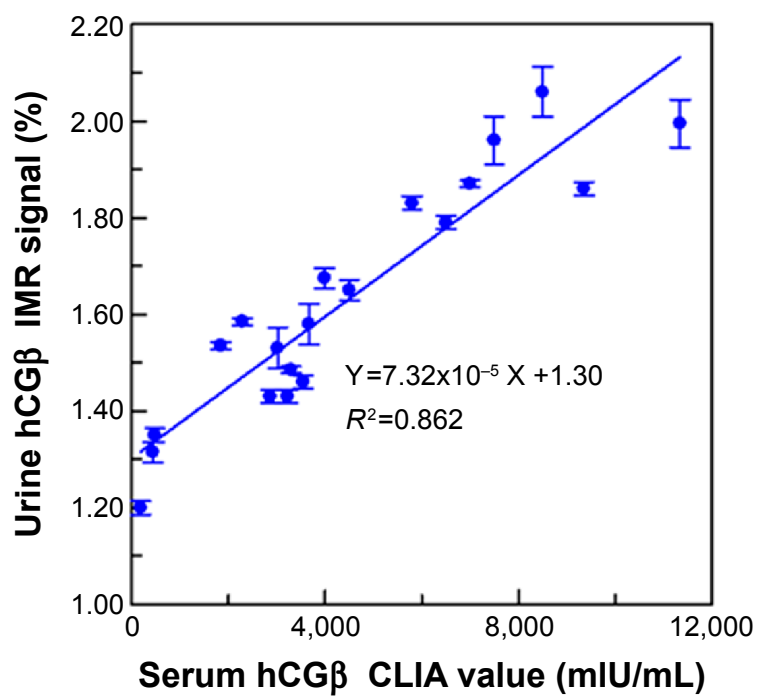

Figure 6 Relationship between urine hCG $\beta$ IMR signals and serum hCG $\beta$ CLIA values $\left(R^{2}=0.862\right)$.

Note: Points represent mean \pm standard deviation.

Abbreviations: hCG $\beta$, total $\beta$-subunit of human chorionic gonadotropin; IMR, immunomagnetic reduction; CLIA, chemiluminescence immunoassay.
The conventional immunochromatographic assay for urine hCG detection is a qualitative test comprising an affinity zone by immobilizing anti-hCG antibodies on a thin porous nitrocellulose membrane to catch targeted hCG in the urine sample. ${ }^{28}$ The cut-off value of commercial assays is $25 \mathrm{mIU} / \mathrm{mL}$. Compared with an immunochromatographic assay for urine hCG detection, there are many benefits of using an IMR assay to detect urine hCG $\beta$. First, the LOD $(0.36 \mathrm{mIU} / \mathrm{mL})$ of the IMR assay was much lower than the cut-off value $(25 \mathrm{mIU} / \mathrm{mL})$ of commercial immunochromatographic assays. Thus, the IMR assay is a potential method due to its much higher sensitivity (69 times higher). Second, as mentioned in the Introduction section, hCG $\beta$ levels are more specific than total hCG levels in pregnancy tests. Therefore, detecting urine hCG $\beta$ with an IMR assay is more specific than with conventional assays to detect urine hCG. Third, the test results of conventional immunochromatographic assays are based on visual interpretation, and thus inter-observer variations in qualitative immunochromatographic assays cannot always be avoided. Quantitative analysis by IMR assays can overcome this drawback.

The conventional ELISA for serum hCG $\beta$ detection is a quantitative test based on two main procedures: (1) using monoclonal antibodies to bind the unique antigenic site on hCG $\beta$ molecules; (2) labeling secondary enzyme-linked antibodies to produce quantitative changes that are directly 
proportional to the amount of targeted hCG $\beta .{ }^{18}$ Furthermore, CLIA employs light-emitting reactions using horseradish peroxidase or alkaline phosphatase as labeling increases the sensitivity and specificity of detection. ${ }^{19}$ Compared with ELISA/CLIA for serum hCG $\beta$ detection, there are also many advantages of using an IMR assay. First, it is easy to operate because no bound/free particle separation processes are involved in the case of an IMR assay; hence, removing magnetic nanoparticles or targeted biomarkers during the assay procedure is not required. Second, it is a homogeneous assay with magnetic signals instead of optical signals, thus avoiding the problem of color intensity decay. Third, the IMR assay is cost-effective because only one monoclonal hCG $\beta$ antibody is used for magnetic reagent synthesis. Fourth, with regards to point-of-care testing, the development of quantitative urine hCG $\beta$ assays for the diagnosis of ectopic pregnancy is essential, and the IMR assay is a potential candidate. In addition, the LOD $(0.36 \mathrm{mIU} / \mathrm{mL})$ of the IMR assay is lower than that $(0.5 \mathrm{mIU} / \mathrm{mL})$ of CLIA (Beckman Coulter), with a high correlation between urine IMR signals and serum CLIA values of hCG $\beta\left(R^{2}=0.862\right)$.

Nevertheless, there are some limitations to this study. First, no interference testing was performed. However, no obvious interfering factors have been reported in the detection of serum biomarkers by IMR assays in previous studies. ${ }^{29,30}$ In the current study, IMR signals for hCG $\beta$ in urine were well correlated to that in serum $\left(R^{2}=0.994\right)$, and thus we did not perform interference testing in this urine IMR assay. Interference in ELISA is primarily due to color change of the substrates that may influence the detection of fluorescence. In addition, the emission intensity of CLIA varies with time, and is influenced by various environmental factors such as temperature, $\mathrm{pH}$, and other materials in the system. Magnetic signals are translucent, and are thus not affected by the sample color. Moreover, the weak connection between nonspecific molecules could be separable in a high-frequency rotating magnetic field. Second, it took nearly 5 hours to complete the real-time magnetic reaction. However, the reaction time could be accelerated to within 30 minutes by properly adjusting laboratory factors, such as reagent concentration, reagent/hCG $\beta$ solution volume, and incubation temperature. ${ }^{31,32}$ Third, the number of clinical samples in this study was limited, and a larger sample size is necessary to support our preliminary results. Furthermore, a high urine hCG $\beta$ IMR signal or serial measurements of increased urine hCG $\beta$ IMR signals are not the only cornerstone of a clinical decision of ectopic pregnancy, and should be combined with transvaginal ultrasound scans. An ectopic pregnancy should be suspected if a high urine hCG $\beta$ IMR signal or serial measurements of increased urine hCG IMR signals without a gestational sac on transvaginal ultrasound scans. On the other hand, serial measurements of decreased urine hCG IMR signals without a gestational sac on transvaginal ultrasound scans are helpful in the clinical diagnosis of an early pregnancy failure.

Prior clinical studies have verified that an IMR assay can be applied to detect biomarkers in serum, plasma, and even cervicovaginal secretions..$^{23,29-31}$ This is the pioneering investigation of using an IMR assay to measure biomarkers in urine, and our findings imply that the IMR assay is a potential candidate for point-of-care testing to assist in the diagnosis of ectopic pregnancy.

\section{Conclusion}

In summary, this study verified that an IMR assay can quantitatively analyze hCG $\beta$ concentrations in clinical urine samples with easy operation, low cost, high sensitivity, and high specificity. Our results show the promising feasibility of assaying urine hCG $\beta$ using IMR to assist in the diagnosis of ectopic pregnancy. A high urine hCG $\beta$ IMR signal or serial measurements of increased urine hCG $\beta$ IMR signals without a gestational sac on transvaginal ultrasound scans in the first trimester should alert clinicians to treat the patient as soon as possible, such as with methotrexate administration to avoid the need of surgical intervention for ruptured ectopic pregnancy and intra-abdominal bleeding, and thus decrease maternal morbidity and mortality.

\section{Acknowledgments}

This work was supported by Mackay Memorial Hospital under grant number MMH-103-46. The authors would like to thank Miss Fang-Ju Sun of the Department of Medical Research of Mackay Memorial Hospital for her guidance on statistical analysis.

\section{Disclosure}

The authors report no conflicts of interest in this work.

\section{References}

1. Marion LL, Meeks GR. Ectopic pregnancy: history, incidence, epidemiology, and risk factors. Clin Obstet Gynecol. 2012;55:376-386.

2. Lozeau AM, Potter B. Diagnosis and management of ectopic pregnancy. Am Fam Physician. 2005;72:1707-1714.

3. Kriebs JM, Fahey JO. Ectopic pregnancy. J Midwifery Womens Health. 2006;51:431-439.

4. Barnhart KT, Katz I, Hummel A, Gracia CR. Presumed diagnosis of ectopic pregnancy. Obstet Gynecol. 2002;100:505-510.

5. Iles RK, Chard T. Molecular insights into the structure and function of human chorionic gonadotrophin. J Mol Endocrinol. 1993;10:217-234. 
6. Ross GT. Clinical relevance of research of the structure of human chorionic gonadotropin. Am J Obstet Gynecol. 1977;129:795-805.

7. Milwidsky A, Adoni A, Miodovnik M, Segal S, Palti Z. Human chorionic gonadotropin (beta-subunit) in the early diagnosis of ectopic pregnancy. Obstet Gynecol. 1978;51:725-726.

8. Shepherd RW, Patton PE, Novy MJ, Burry KA. Serial beta-hCG measurements in the early detection of ectopic pregnancy. Obstet Gynecol. 1990;75:417-420.

9. Dart RG, Mitterando J, Dart LM. Rate of change of serial beta-human chorionic gonadotropin values as a predictor of ectopic pregnancy in patients with indeterminate transvaginal ultrasound findings. Ann Emerg Med. 1999;34:703-710.

10. Hackshaw AK, Wald NJ. Assessment of the value of reporting partial screening results in prenatal screening for Down syndrome. Prenat Diagn. 2001;21:737-740.

11. Casart YC, Camejo MI, Proverbio F, Febres F. Bioactivity of serum hCG in preeclampsia. Obstet Gynecol. 2001;98:463-465.

12. Harvey RA, Mitchell HD, Stenman UH, et al. Differences in total human chorionic gonadotropin immunoassay analytical specificity and ability to measure human chorionic gonadotropin in gestational trophoblastic disease and germ cell tumors. J Reprod Med. 2010;55:285-295.

13. Lohstroh PN, Overstreet JW, Stewart DR, et al. Secretion and excretion of human chorionic gonadotropin during early pregnancy. Fertil Steril. 2005;83:1000-1011.

14. Chung K, Allen R. The use of serial human chorionic gonadotropin levels to establish a viable or a nonviable pregnancy. Semin Reprod Med. 2008;26:383-390.

15. Visconti K, Zite N. hCG in ectopic pregnancy. Clin Obstet Gynecol. 2012;55:410-417.

16. Mehta TS, Levine D, Beckwith B. Treatment of ectopic pregnancy: Is a human chorionic gonadotropin level of $2,000 \mathrm{mIU} / \mathrm{mL}$ a reasonable threshold? Radiology. 1997;205:569-573.

17. Mol BW, Hajenius PJ, Engelsbel S, et al. Serum human chorionic gonadotropin measurement in the diagnosis of ectopic pregnancy when transvaginal sonography is inconclusive. Fertil Steril. 1998;70:972-981.

18. Lequin RM. Enzyme immunoassay (EIA)/enzyme-linked immunosorbent assay (ELISA). Clin Chem. 2005;51:2415-2418.

19. Vankrieken L, De Hertogh R. Rapid, automated quantification of total human chorionic gonadotropin in serum by a chemiluminescent enzyme immunometric assay. Clin Chem. 1995;41:36-40.
20. Yang SY, Jian ZF, Chieh JJ, et al. Wash-free, antibody-assisted magnetoreduction assays on orchid viruses. $J$ Virol Methods. 2008;149: 334-337.

21. Chen CY, Chang CC, Yu C, Yang SY, Lin CW. Immunomagnetic reduction for fetal fibronectin detection-a novel method to detect the preterm biomarker. Biomed Eng App Bas C. 2011;23:273-278.

22. Jiang WQ, Yang HC, Yang SY, et al. Preparation and properties of superparamagnetic nanoparticles with narrow size distribution and biocompatible. J Magn Magn Mater. 2004;283:210-214.

23. Chen CY, Chang CC, Lin CW. Clinical application of immunomagnetic reduction for quantitative measurement of insulin-like growth factor binding protein-1 in the prediction of pregnant women with preterm premature rupture of membranes. Clin Chim Acta. 2015;438:337-341.

24. Barnhart KT. Clinical practice. Ectopic pregnancy. $N$ Engl J Med. 2009;361:379-387.

25. Gracia CR, Barnhart KT. Diagnosing ectopic pregnancy: decision analysis comparing six strategies. Obstet Gynecol. 2001;93:464-470.

26. Renna MD, Pisani P, Conversano F, et al. Sonographic markers for early diagnosis of fetal malformations. World J Radiol. 2013;5:356-371.

27. Armbruster DA, Tillman MD, Hubbs LM. Limit of detection (LQD)/ limit of quantitation (LOQ): comparison of the empirical and the statistical methods exemplified with GC-MS assays of abused drugs. Clin Chem. 1994:40:1233-1238.

28. Osikowicz G, Beggs M, Brookhart P, et al. One-step chromatographic immunoassay for qualitative determination of choriogonadotropin in urine. Clin Chem. 1990;36:1586.

29. Huang KW, Yang SY, Hong YW, et al. Feasibility studies for assaying alpha-fetoprotein using antibody-activated magnetic nanoparticles. Int J Nanomedicine. 2012;7:1991-1996.

30. Chang CH, Lai ZX, Lin HL, et al. Use of immunomagnetic reduction for C-reactive protein assay in clinical samples. Int J Nanomedicine. 2012;7:4335-4340.

31. Chiu MJ, Yang SY, Horng HE, et al. Combined plasma biomarkers for diagnosing mild cognition impairment and Alzheimer's disease. ACS Chem Neurosci. 2013;4:1530-1536.

32. Yang SY, Chang JF, Chen TC, Yang CC, Ho CS. Study of the temperature dependent immuno-reaction kinetics for the bio-functionalized magnetic nanoparticle assay of bio-markers of colorectal cancer. Appl Phys Lett. 2014;104:013702.
International Journal of Nanomedicine

\section{Publish your work in this journal}

The International Journal of Nanomedicine is an international, peerreviewed journal focusing on the application of nanotechnology in diagnostics, therapeutics, and drug delivery systems throughout the biomedical field. This journal is indexed on PubMed Central,

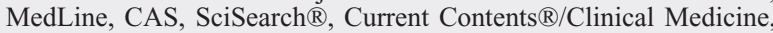

\section{Dovepress}

Journal Citation Reports/Science Edition, EMBase, Scopus and the Elsevier Bibliographic databases. The manuscript management system is completely online and includes a very quick and fair peer-review system, which is all easy to use. Visit http://www.dovepress.com/ testimonials.php to read real quotes from published authors. 